\title{
Pancreatic Epithelial Cells Form Islet-Like Clusters in the Absence of Directed Migration
}

\author{
Steven J. Holfinger, ${ }^{1}$ James W. Reinhardt, ${ }^{1}$ Rashmeet Reen, ${ }^{1}$ Kevin M. Schultz, ${ }^{2}$ Kevin M. Passino, ${ }^{2}$ \\ William E. Ackerman, ${ }^{3}$ Douglas A. Kniss, ${ }^{1,3}$ Leonard M. Sander, ${ }^{4}$ Daniel Gallego-Perez, ${ }^{1}$ \\ and KeITH J. GoOCH ${ }^{1,5}$
}

\begin{abstract}
${ }^{1}$ Department of Biomedical Engineering, College of Engineering, The Ohio State University, Columbus, OH 43210, USA; ${ }^{2}$ Department of Electrical and Computer Engineering, The Ohio State University, Columbus, OH 43210, USA; ${ }^{3}$ Department of Obstetrics and Gynecology, College of Medicine, and Wexner Medical Center, The Ohio State University, Columbus, OH 43210, USA; ${ }^{4}$ Department of Physics, University of Michigan, Ann Arbor, MI 48109, USA; and ${ }^{5}$ The Dorothy M. Davis Heart and Lung Research Institute, The Ohio State University, 270 Bevis Hall 1080 Carmack Rd., Columbus, OH 43210, USA
\end{abstract}

(Received 5 December 2014; accepted 9 May 2015; published online 22 May 2015)

Associate Editor Michael R. King oversaw the review of this article.

\begin{abstract}
The endocrine differentiation of pancreatic ductal epithelial cells is dependent upon their transition from a twodimensional monolayer to three-dimensional islet-like clusters. Although clustering of these cells is commonly observed in vitro, it is not yet known whether clustering results from long-range signaling (e.g., chemotaxis) or short-range interactions (e.g., differential adhesion). To determine the mechanism behind clustering, we used experimental and computational modeling to determine the individual contributions of long-range and short-range interactions. Experimentally, the migration of PANC-1 cells on tissue culture treated plastic was tracked by time-lapse microscopy with or without a central cluster of cells that could act as a concentrated source of some long-range signal. Cell migration data was analyzed in terms of distance, number of steps, and migration rate in each direction, as well as migration rate as a function of distance from the cluster. Results did not indicate directed migration toward a central cluster $(p>0.05)$. Computationally, an agent-based model was used to demonstrate the plausibility of clustering by short-range interactions only. In the presence of random cell migration, this model showed that a high, but not maximal, cell-cell adhesion probability and minimal cell-substrate adhesion probability supported the greatest islet-like cluster formation.
\end{abstract}

Keywords-Diabetes, Islet cells, Differential adhesion, Agent-based modeling, Time-lapse microscopy.

Address correspondence to Keith J. Gooch, The Dorothy M. Davis Heart and Lung Research Institute, The Ohio State University, 270 Bevis Hall 1080 Carmack Rd., Columbus, OH 43210, USA. Electronic mail: gooch.20@osu.edu

Steven J. Holfinger and James W. Reinhardt are co-first authors.

\section{INTRODUCTION}

Type I diabetes mellitus, also called insulin-dependent and juvenile diabetes, is an autoimmune disease characterized by the destruction of insulin-secreting beta cells of the pancreas. This disease afflicts approximately one million individuals in the U.S. ${ }^{13}$ and is increasing in incidence worldwide at an annual rate of $3-5 \% .{ }^{19}$ Type I diabetes is traditionally managed by exogenous insulin therapy, but this method has limited ability to tightly regulate blood glucose levels and often results in long-term complications. Alternative treatments that may improve long-term outcomes include whole pancreas or pancreatic islet cell transplantation, but the annual supply of donor organs is severely limited and therefore reserved only for severe cases. ${ }^{18,21,33}$ To fill the unmet need for insulin producing cells and improve long-term efficacy of treatment, researchers have explored alternative sources of insulin-secreting cells that include the self-replication of beta cells, differentiation of progenitor or stem cells, and transdifferentiation of related cell types toward a pancreatic endocrine phenotype..$^{2,5,12,27,30}$ The upregulation of islet cell hormone genes (e.g., insulin, glucagon, c-peptide, and somatostatin) and their secretion are associated with clustering and the transition from a two-dimensional monolayer to three-dimensional islet-like structures in vitro..$^{5-7,24,37}$ Further supporting the importance of the $3 \mathrm{D}$ structure is the observation that the secretory responsiveness of cells in 3D clusters was lost upon dispersal of the cells and established again after $3 \mathrm{D}$ isletlike structures were reformed. ${ }^{24}$

Of the various cell types explored as a source of new beta cells, pancreatic ductal epithelial cells (PDEC) are 
attractive candidates. ${ }^{4-6}$ During the normal development of the pancreas, islet precursor cells initially bud off of the ductal structures with evidence of transdifferentiation of these PDEC to endocrine cells. ${ }^{29,31}$ PANC-1 cells are human epithelial-like adenocarcinoma cells that have been used as a model system for PDECs because of this pancreatic ductal origin. ${ }^{14}$ This cell line clusters when plated on culture dishes and exposed to serum-free medium (SFM), and later shows signs of endocrine differentiation by secreting C-peptide, insulin, and glucagon. ${ }^{17}$

Little is known about how PANC-1 cells form multicellular clusters. Possible mechanisms can be broadly categorized into those relying on long-range or shortrange interactions. Cell clustering by long-range interactions involves some signal for mutual attraction followed by directed migration of cells toward one another. Perhaps the best-studied example of a longrange signal mediating cell clustering is chemotaxis by Dictyostelium discoideum. ${ }^{35}$ These single-cell organisms release cAMP, collectively forming cAMP gradients, then aggregate by migrating in the direction of increasing cAMP concentration. Chemotaxis is not a behavior unique to $D$. discoideum, but has also been suggested as a mechanism for directed migration for Escherichia coli ${ }^{16,38}$ and in mammalian cell culture. ${ }^{22,26}$ Based on observations that PANC-1 cells in a Boyden chamber migrated toward a source of fibroblast growth factor two (FGF2) and that interfering with FGF2 signaling blocked aggregation, Hardikar et al. asserted that PANC-1 cells use FGF2 as a paracrine chemoattractant to mediate clustering. ${ }^{17}$ In addition to this type of chemical signaling, mechanical cues may direct longrange cell clustering as well. Cells on or in an extracellular matrix can mechanically remodel the surrounding matrix. ${ }^{10,36}$ The resulting anisotropic changes in matrix density, matrix mechanics, and the alignment of fibrous proteins $^{25}$ have all been proposed as mechanisms by which cells can direct their migration toward other cells via haptotaxis, mechanosensing, or contact guidance. ${ }^{25,36,40}$ In contrast to long-range interactions, cell clustering could also result from short-range interactions whereby cells migrate randomly, incidentally collide, and intercellular adhesions form causing cells to stick together. Boretti and Gooch suggested that shortrange mechanical interactions regulate the clustering of primary human pancreatic epithelial cells based on their observation that the relative strength of cell-cell vs. cellsubstrate adhesion was a primary regulator of clustering. ${ }^{5}$ This concept is akin to the "differential adhesion hypothesis" that explains cell migration and sorting during tissue morphogenesis. ${ }^{34}$

In the present study, we investigate whether clustering of PANC-1 cells in vitro shows evidence longrange signaling and whether differential adhesion alone can support the formation of cell clusters in computational models. We seek to understand the mechanisms behind pancreatic cell clustering. This understanding will facilitate informed decisions about how to control clustering of pancreatic cells, improving differentiation toward a secretory endocrine phenotype, and likely have implications for various other cell types whose functions are dependent upon multicellular aggregation such as hepatocytes ${ }^{15,23}$ and embryonic stem cells. ${ }^{1,8,20,28}$

\section{MATERIALS AND METHODS}

\section{Cell Culture and Clustering}

PANC-1 cells (American Type Culture Collection, Manassas, VA) were cultured in serum-containing medium (SCM) consisting of low-glucose Dulbecco'smodified Eagle medium (DMEM) with 10\% fetal bovine serum (FBS, Gibco/Invitrogen, Carlsbad, CA). Cell clustering was induced by changing medium to SFM consisting of a 1:1 mixture of DMEM and F12 supplemented with $1 \%(\mathrm{v} / \mathrm{v})$ insulin transferrin selenium solution (ITS, Gibco/Invitrogen, Carlsbad, CA) and 1\% (w/ v) bovine serum albumin (MP Biomedicals, Solon, $\mathrm{OH}$ ).

\section{Hanging-Drop Method for Forming PANC-1 Cell Clusters}

The hanging drop method was used to create preformed clusters for experiments requiring initial cell aggregates. When PANC-1 cells were initially seeded in SCM, 45 aliquots $(4 \mu \mathrm{L}$ each) of cell-containing SFM were pipetted onto the lid of a $100 \mathrm{~mm}$ tissue culture treated plastic (TCTP) Petri dish (Fisher Scientific, Pittsburgh, PA). Each $4 \mu \mathrm{L}$ drop contained approximately $\sim 40$ cells for cultures at $10 \%$ confluence, and $\sim 20$ cells for cultures at $5 \%$ confluence. The dish was then inverted and incubated for 16-20 h. Immediately before time-lapse imaging, cell clusters that formed in the hanging drops were washed off the lid with $1 \mathrm{~mL}$ SFM and added to the $35 \mathrm{~mm}$ Petri dish containing PANC-1 cells at 10 or $5 \%$ confluence.

\section{Time-Lapse Microcopy and Image Analysis}

Cells were seeded onto $35 \mathrm{~mm}$ TCTP petri dishes (MatTek, Ashland, MA) in SCM at $\sim 10 \%$ confluence (75,000 cells/dish) or $\sim 5 \%$ confluence $(37,500$ cells/ dish) and cultured for 16-20 h. After this culture period, culture medium was changed to SFM to allow for cell clustering and $1 \mathrm{~mL}$ of SFM containing PANC-1 cell clusters was added. Although the hanging-drop method could potentially produce up to 45 clusters, fewer than 10 clusters were typically observed 
after their transfer to the Petri dish. If the experiment contained clusters, a field of view was chosen such that: (1) contained a cluster of the intended size (i.e., $~ 20$ or $\sim 40$ cells), (2) there were no other clusters within $\sim 5000 \mu \mathrm{m}$, and (3) the local confluence of PANC-1 cells was representative of the desired confluence (i.e., $\sim 5$ or $\sim 10 \%$ ). All images were taken of a single field of view using differential interference contrast microscopy with an Axiovert $200 \mathrm{M}$ inverted microscope (Zeiss, Thuringia, Germany) equipped with a $\times 10$ objective. The cells and microscope were housed in a Marianas microscope enclosure that was maintained at $37{ }^{\circ} \mathrm{C}$ and $5 \% \mathrm{CO}_{2}$ and rested on a vibration isolation table. Images were taken every five min for $25 \mathrm{~h}$ and captured using SlideBook software (3i Intelligent Imaging Innovations, Denver, CO).

\section{Analysis of Pancreatic Cell Migration}

The Manual Tracking plug-in (Fabrice P. Cordelières, Institut Curie, Orsay, France) for ImageJ (W. Rasband, NIH) was used to identify the coordinates of the center of the cluster and surrounding cells every five min. All data were recorded by the same person. Cells were temporarily not tracked if they migrated outside the field of view. Data from cells that appeared to undergo apoptosis during the recorded time frame were discarded. Small clusters, comprised of more than two cells, made up less than $3 \%$ of cell tracks. Data from the movement of these small clusters was excluded from analyzes because they were not as motile single or joined pairs of cells. Individual cell tracks were then imported into MATLAB (MathWorks, Natick, MA) and analyzed using original code found in the Supporting Material. This code allowed for the full data set to be analyzed, or subsets of data demarcated by one of a variety of parameters including the number of cells (e.g., single cells, pairs of attached cells, etc.), cell fate, and the selection of a specific time step. For a given time step, MATLAB determined $\Theta$, the direction of cell migration relative to the cluster (Fig. 1e), as well as L, the distance migrated during a single time step. The path length for a single cell was calculated as the sum of all distances traveled each time step. Data for distances traveled and steps were sorted into bins, each bin representing movement in a particular direction in relation to the cluster. A step represents all movement in any direction for a single cell during a single time step. This is distinct from a time step, the length of time between data points. The binned data was then used to generate rose diagrams. Migration rate within each bin was calculated by dividing the total distance traveled by cells in that direction by the number of steps taken in that direction. Cell migration data were combined from three inde- pendent experiments $(n=3)$ for each condition: $10 \%$ confluence with a cluster (171 cell tracks), 10\% confluence without a cluster (232 cell tracks), and 5\% confluence with a cluster (165 cell tracks).

\section{Agent-Based Simulations}

A more complete description of the rules of the agent-based model, conditions simulated, and analysis of simulation results can be found in the Supporting Material. Briefly, agent-based modeling conducted in NetLogo ${ }^{39}$ was used to simulate the in vitro clustering of cells on a 2D substrate and explore the feasibility of cell clustering in the absence of long-range interactions. The in silico world in which this model exists consists of a $50 \times 50 \times 7$ arrangement of patches, each of which is a unit cube with dimensions equaling the diameter of a cell $(20 \mu \mathrm{m})$, and wraps in the XY plane. Substrate was only modeled on the base layer. Cells were represented as individual spherical agents, each centered in a unit cell, and possessed a limited number of available actions regulated by strictly defined rules. These actions included binding to other cells, breaking bonds with other cells and moving within the 3D tetragonal simulation space. Cells could form up to six bonds, one at each of its four in-plane sides and one at each of its two out-of-plane sides.

All simulations were run at $40 \%$ confluence, a value chosen to help distinguish conditions that support clustering and those that do not (see Supporting Material for detailed explanation of parameter choice). The probability a cell would form a bond with a neighboring cell was equal to the cell-cell adhesion probability $(\%)$. The probability a cell would unbind was equal to $100 \%$ minus the cell-cell adhesion probability. Therefore, both the probability of binding and unbinding add to $100 \%$. cell-substrate adhesion probability (\%) was another variable in the model. Similar to cell-cell adhesion, cell substrate adhesion and de-adhesion probabilities sum to $100 \%$. Cells adhered to the substrate were restricted from climbing from the base level to the second level. Although cell-substrate adhesion probability may in reality affect other cell behaviors such as migration, this coupling was not included in the model. Cell-cell adhesion probability was explored at seven levels $(0,10,30$, $50,70,90$, and $99 \%$ ) and cell-substrate adhesion probability at seven levels $(0,10,30,50,70,90$, and $100 \%)$ for a total of 49 unique combinations. Ten independent simulations were run for each combination. A cell-cell adhesion probability of $100 \%$ was not evaluated as the cells would have rapidly bound together and been unable to unbind or move thereafter. The probability of movement had a moderate influence on cluster size and density, but was not explored systematically for these experiments and was set arbitrarily at $50 \%$. 
All models were run for 15,000 iterations, a sufficient duration for all conditions to reach equilibrium. One iteration represents the application of all relevant rules to all cells. Equilibrium was chosen as the endpoint because different simulations approached equilibrium at different rates. By ensuring all simulations had reached equilibrium, relative differences between results would not be simulation time dependent. All rules in our model were probabilistic, and the order of the cells to which the rules were applied each iteration was random. Therefore, even simulations with identical initial conditions almost certainly had different outcomes. Three approaches were used to quantify the degree of clustering: (1) average cluster size (cells/cluster), (2) average height, and (3) compactness. Average height provides a measure of three-dimensionality and was calculated by dividing the total number of cells in a cluster by its footprint (i.e., area in patches a cluster occupies on the base level). Compactness describes how efficiently the cells are packed and is defined in detail in the Supporting Material. Even for simulations at equilibrium, measured average height and compactness varied moderately with time, and therefore the average values for the final 1000iteration range (i.e., iteration 14,001-15,000) were recorded. Average cluster size was more stable with time and was calculated after the final iteration. Only data from simulations that had reached equilibrium are reported here, the criteria for which is described in detail in the Supporting Material.

\section{Statistics}

Statistical analysis was performed using SPSS v. 22 (SPSS, Chicago, IL). Significance was determined using a one-sample $t$ test. Bonferroni correction was used to adjust the $p$ value for multiple comparisons. Error bars shown represent the standard deviation or $95 \%$ confidence interval. Rose diagrams were generated in MATLAB and mean resultant vectors were calculated using the circular statistics (directional statistics) toolbox. $^{3}$ The mean resultant vector is the sum of a collection of vectors, each with a magnitude and direction. The magnitude of the mean resultant vector is presented as a percentage of the sum of the magnitudes of its constituent vectors.

\section{RESULTS AND DISCUSSION}

\section{PANC-1 Cells Do Not Exhibit Preferential Migration Toward Cell Clusters}

Consistent with previous observations, ${ }^{5-7,12,24,37}$ PANC-1 cells at and near confluence formed $3 \mathrm{D}$ multicellular clusters when cultured in SFM (Supplementary Movie S1). In an attempt to explain the sixfold increase in migration rate and subsequent clustering of pancreatic cells upon the removal of serum, Hardikar et al. suggested that serum could contain "inhibitory factors" that restrict cell migration. ${ }^{17}$ Though not directly inhibitory, serum contains proteins (e.g., albumin and fibronectin) that can adsorb onto TCTP and alter the adhesiveness of the surface. ${ }^{9}$ Exchanging the SCM for SFM may alter the composition of adsorbed proteins and could reduce its adhesiveness. A less adhesive surface might allow cells to migrate more freely, increasing the opportunity for cells to contact each other and form clusters. However, it is not known to what extent removal of serum alters the likelihood and stability of intercellular adhesions. The high cell densities used for Hardikar's experiments, however, are not practical for testing the hypothesis that clustering results from long-range mutual attraction between cells. This is because cells start out in close proximity to, and sometimes in contact with, several cells. Cell migration and the unknown effect of cell-cell distance on long-range signaling add further complexity. As a result, the direction in which a cell would be expected to move cannot be easily or adequately predicted based on the location of multiple migrating neighbors. To simplify this system: (1) the initial seeding density was reduced to $\sim 10 \%$ confluence and (2) a preformed cluster of PANC-1 cells was added to act as a concentrated source of any long-range signal that might exist. If PANC-1 cells cluster by long-range signaling, individual cells would be expected to migrate preferentially toward the preformed cluster. This same experimental design without a preformed cluster was used as a control. The positions of individual PANC-1 cells, pairs of joined cells, and the central cluster were then tracked in five min intervals for $25 \mathrm{~h}$ (Fig. 1). For experiments without a central cluster, an arbitrary central point was used as a reference point for the migration of cells. Movement was tracked in relation to the center of the central cluster at the time of measurement, not in relation to the edge of the cluster. Thus, data was not adjusted due changing size or shape as well as modest migration of the cluster. For each condition, the total distance all tracked cells migrated in different directions relative to the cluster was plotted as a rose diagram and used to calculate a mean resultant vector (Figs. 2a and 2b). The magnitude and direction of the mean resultant vector were used to assess whether cells exhibited preferential migration. Specifically, the projection of the mean resultant vector onto the unit vector toward the cluster, or dot product, represents the overall percentage of migration toward the cluster. Since there do not exist established criteria 


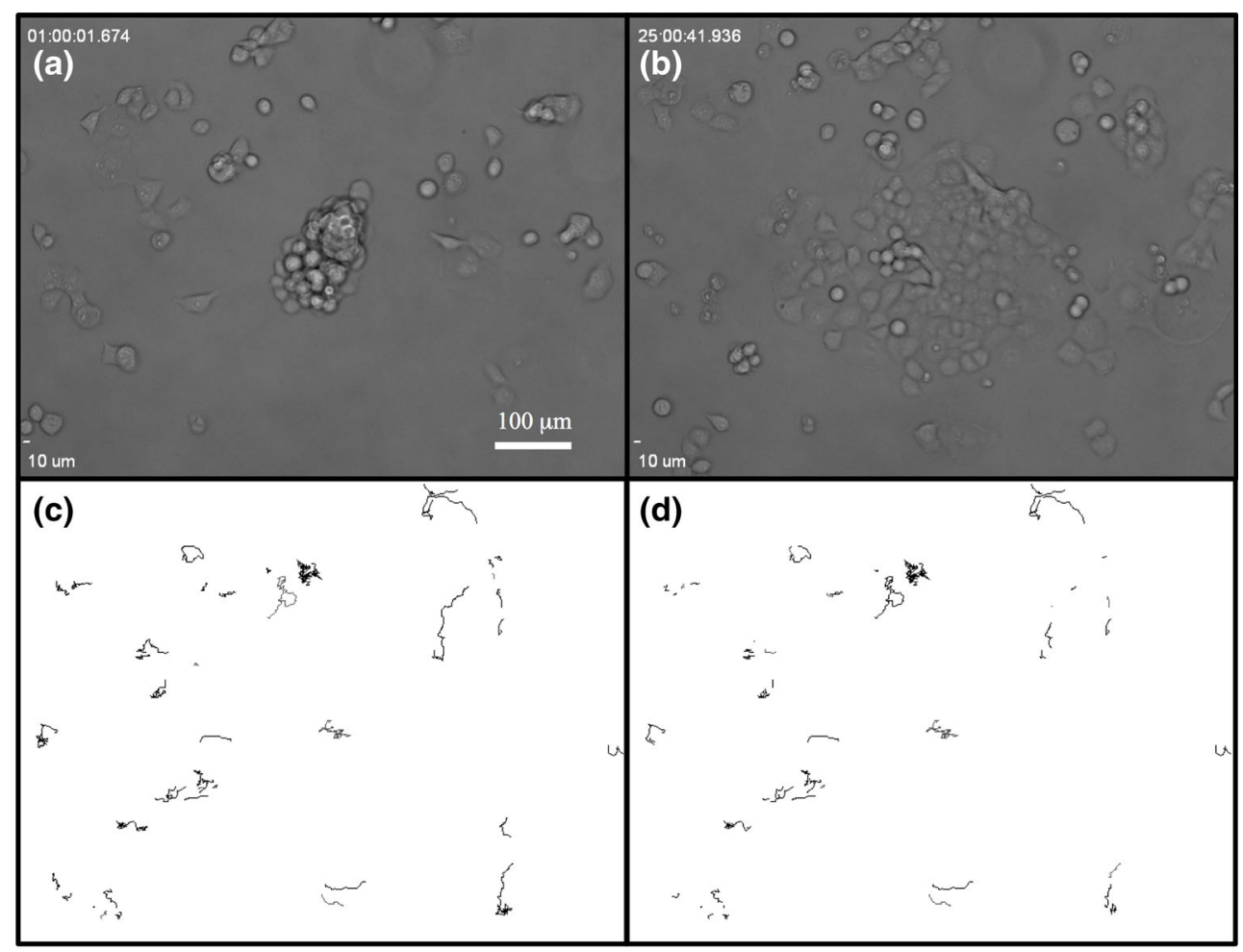

(e)

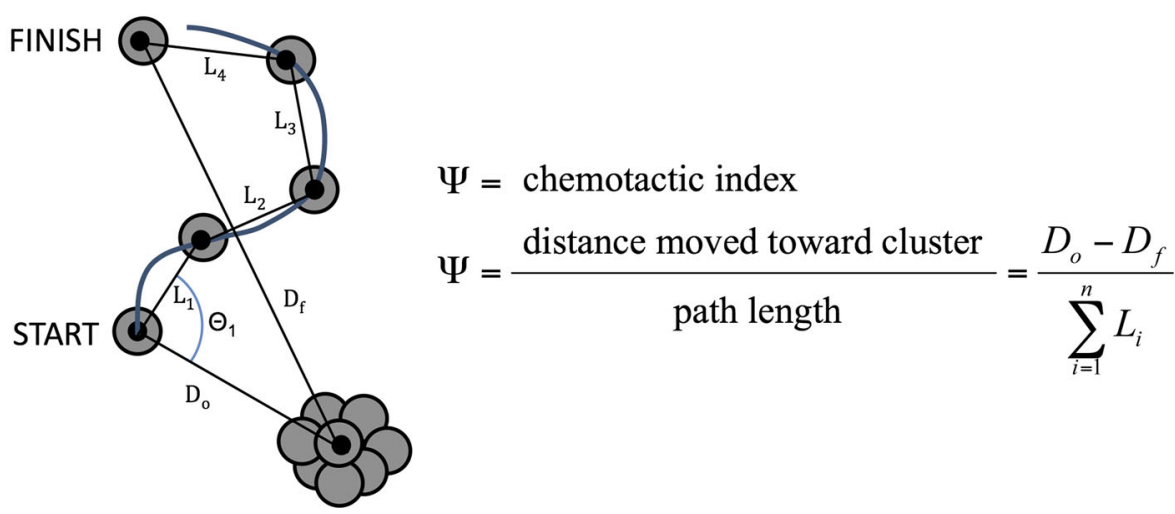

FIGURE 1. Tracking PANC-1 cell migration. PANC-1 cells cultured at $10 \%$ confluence with a central cluster imaged at $0 \mathrm{~h}$ (a) and after $24 \mathrm{~h} \mathrm{(b).} \mathrm{(c)} \mathrm{The} \mathrm{corresponding} \mathrm{cell} \mathrm{tracks} \mathrm{throughout} \mathrm{this} \mathrm{time} \mathrm{period.} \mathrm{(d)} \mathrm{The} \mathrm{same} \mathrm{cell} \mathrm{tracks} \mathrm{after} \mathrm{applying} \mathrm{a} \mathrm{motility} \mathrm{filter.}$ (e) Illustrative example depicting how the chemotactic index is calculated. The straight solid black lines $\left(L_{1}-L_{4}\right)$ represent the displacement recognized by the MATLAB code during each time step. $D_{0}$ and $D_{\mathrm{f}}$ represent the initial and final distance to the cell cluster, respectively. During the first time step the cell moves a straight-line distance $L_{1}$ at an angle $\Theta_{1}$ with respect to the cluster. Subsequent steps are labeled $L_{2}-L_{4}$. The chemotactic index is calculated by dividing the change in distance to the cluster $\left(D_{\mathrm{f}}-D_{0}\right)$ by the path length traveled $\left(\sum L_{1-4}\right)$.

for determining critical values for statistical significance in directional data, we have chosen $1 \%$ overall migration toward a cluster. To clarify the results reported below, the magnitude of the mean resultant vector is reported within parentheses, not the overall percentage of migration towards the cluster. For this initial experiment, the mean resultant vector did not indicate preferential migration toward the cluster and in fact showed that there was greater net migration away from the cluster $(5.88 \%)$ than away from an arbitrary central point in the control experiments $(1.30 \%)$. One possibility for not detecting directed migration toward the cluster is that for a given cell, directional signals from nearby cells might mask the influence of the more distant cluster. To guard against this possibility and reduce the potential masking effect of nearby cells the seeding density was reduced further to $\sim 5 \%$ confluence (Supplementary Movie S2). Still, at this reduced density, net migration toward the cluster was not detected (Fig. 2c). A similar 
TABLE 1. Mean chemotactic index values.

\begin{tabular}{llll}
\hline Analysis & \multicolumn{1}{c}{ Experiment } & Mean \pm SD & $p$ value \\
\hline All Data & $10 \%$ confluence & $-0.036 \pm 0.337$ & 0.172 \\
& $10 \%$ confluenceNo cluster & $-0.006 \pm 0.276$ & 0.753 \\
Motility Filter & $5 \%$ confluence & $-0.028 \pm 0.310$ & 0.245 \\
& $10 \%$ confluence & $-0.045 \pm 0.479$ & 0.149 \\
& $10 \%$ confluenceNo cluster & $-0.020 \pm 0.441$ & 0.428 \\
30 Min Intervals & $5 \%$ confluence & $-0.034 \pm 0.485$ & 0.277 \\
& $10 \%$ confluence & $-0.067 \pm 0.429$ & 0.052 \\
& $10 \%$ confluenceNo cluster & $-0.006 \pm 0.374$ & 0.806 \\
& $5 \%$ confluence & $-0.030 \pm 0.397$ & 0.330 \\
\hline
\end{tabular}

analysis was performed examining total number of steps (Figs. 2d-2f) and the average migration rate (Figs. 2g-2i) in different directions relative to the cluster. Average migration rate was calculated by dividing the distance traveled in a given direction by the total number of steps taken in that same direction. Only for average migration rate did the mean resultant vector point toward the cluster (Figs. $2 \mathrm{~h}$ and $2 \mathrm{i}$ ), yet the magnitude was small $(<1 \%)$ and therefore not strongly suggestive of directed migration.

The most straightforward interpretation of these data is that long-range signals are not involved. However, the mean resultant vector is only one metric for this rich set of data and not adequately rigorous to support broad conclusions about the presence/absence of preferential migration. One potentially important parameter ignored by the mean resultant vector method is the effect of a cell's distance from the cluster, a potential source of chemoattractant. For example, if a cell were too close to a source of chemoattractant the cell's receptors could become saturated and the cell would lose its ability to sense the gradient. ${ }^{16,38}$ Alternatively, if a cell were too far from the cluster the concentration of the chemoattractant could be too low for the cell to detect. To visualize the effect of distance from the cluster on cellular migration rate toward the cluster, we broke down each cell track into its individual steps, then segregated these steps by the cell's distance from the cluster at the beginning of the step (Fig. 3). These segregated steps were binned in $50 \mu \mathrm{m}$ intervals up to the edge of our field of view, $600 \mu \mathrm{m}$ from the center of the cluster. Since clusters were roughly $100 \mu \mathrm{m}$ in diameter, cells could not be tracked within $50 \mu \mathrm{m}$ from the center of clusters. When accounting for multiple comparisons using Bonferroni correction, none of the results achieved statistical significance $(p>0.05)$. However, because Bonferroni correction is highly conservative and due to the high number of comparisons (i.e., 21), correcting for multiple comparisons might hide truly significant results. Therefore, we have also chosen to present the data without correcting for multiple comparisons. In six cases average migration rate was away from the central cluster $(p<0.05)$. Interestingly, four of these six cases were for cells at $10 \%$ confluence between 100 and $300 \mu \mathrm{m}$ from the central cluster. For only one condition, $5 \%$ confluence with a central cluster, and for cells between 350 and $400 \mu \mathrm{m}$ away from the cluster, was average migration rate toward the cluster significant $(p=0.013)$.

We then analyzed our data by calculating the chemotactic index, also referred to as the McCutcheon index, a measurement that has been used previously to reveal collective cell migration toward a chemotactic source. ${ }^{22,26,35}$ This index was calculated for an individual cell by dividing the cell's change in distance from the cluster by its path length (Fig. 1e). The result is a value between +1 and -1 , where +1 signifies all movement was directly toward the cluster, -1 signifies all movement was directly away from the cluster, and 0 signifies that though the cell may have moved, it ended up the same distance from the cluster as when tracking was started. Values for the chemotactic index of individual cells (Fig. 4) conformed to a bell-shaped distribution. In all cases, collective migration with respect to the cluster was not significant $(p>0.05)$ (Table 1).

Next, we carefully dissected our methods to assess aspects that might influence the quality of our data and potentially impact our findings. Empirical testing of the reproducibility of manually tracking cells suggested that variability in the assignment of cell position could be up to several microns. Considering the actual distance and direction of cell migration as the signal, this variability would represent noise that could impact the measured distance and direction of cell migration. This noise would be especially pronounced if the distance moved by a cell during an interval was small and could mask preferential migration toward a cluster. To increase the signal-to-noise ratio, two strategies were used: (1) applying a threshold to remove cell migration below $10 \mu \mathrm{m}$ in a 60 min period and (2) using longer, $30 \mathrm{~min}$ intervals. Applying this threshold removed $\sim 60 \%$ of cells steps; however, it appeared to preserve most of the steps in which cells migrated (Figs. 1c and 

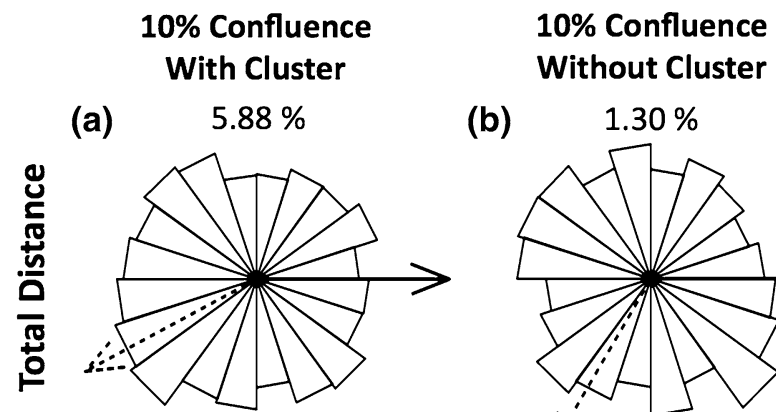

(b)

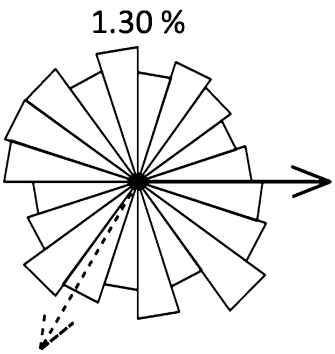

(d)

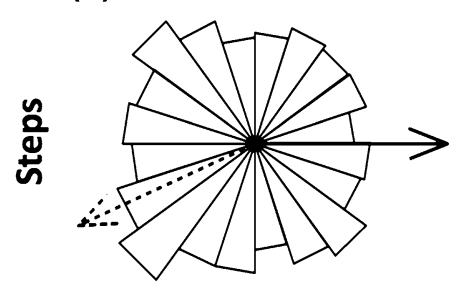

(g)

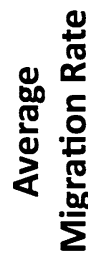

(e)

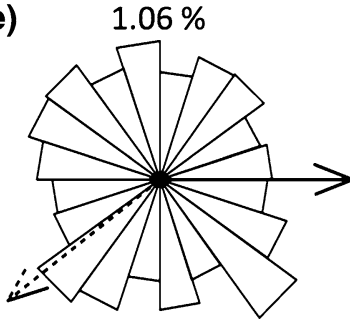

\section{5\% Confluence \\ With Cluster}

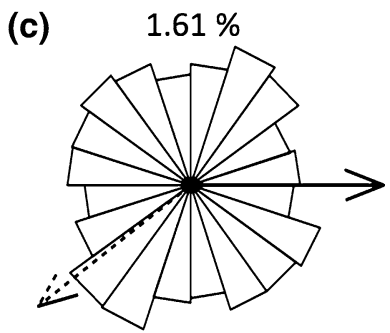

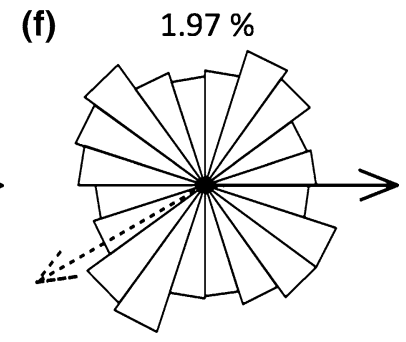

(i)

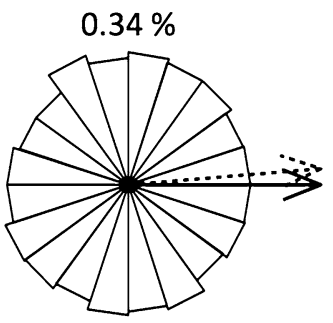

FIGURE 2. Polar histograms of cell migration data. Data was analyzed by total distance migrated (a-c), total number of steps (df), or average migration rate ( $g-i)$ in relation to the central cluster or in relation to an arbitrary central point if there was no cluster. The direction toward the central cluster (solid arrow) and mean resultant vector (dashed arrow) are overlain on each figure. For experiments without a central cluster, the solid arrow points to an arbitrary central point. The magnitude of the mean resultant vector is included in each panel (range 0-100\%).

1d). Mean chemotactic indices were calculated after applying both of these strategies and did not reveal the presence of preferential migration (Table 1). Rose diagrams corresponding to this data are available in the Supporting Material (Fig. S1).

Some analyzes revealed statistically significant migration away from the cluster. These findings have a few possible explanations. First, over the course of experiments clusters gradually flattened (Figs. 1a and b) and some cells separated then migrated away (Supplementary Movie S3). The tracking of these cells was no different from other cells, although these cells were few. Second, for cells that do not bind to the cluster, the cluster might obstruct the migration of cells, thus biasing subsequent migration away from the cluster. This might explain migration away from the cluster for cells at 5\% confluence between 50 and $100 \mu \mathrm{m}$ from the cluster (Fig. 3). Third, migration away from a cluster may indicate a rarely observed phenomenon termed fugetaxis. Instead of producing chemoattractant, cells in a formed cluster may produce chemorepellant, perhaps for the purpose of limiting cluster size. An additional concern is the presence of spontaneously formed small clusters that might act as additional sources of some long-range signal and influence the migration of nearby cells. However, because the number of these small clusters is few $(<3 \%)$ and their size small relative to the central cluster we would not expect their presence to prevent the detection of directed migration.

\section{Agent-Based Modeling Suggests Cell Clustering Might Result from Short-Range Interactions}

The balance between cell-substrate and cell-cell adhesiveness, or differential adhesion, was proposed as 
a potential regulator of cell clustering. We developed an agent-based computational model to assess this idea. In the model, simulated cells could migrate, bind to the substrate, and bind to each other. Importantly, in this model there is no mechanism for long-range cell-cell communication. Cell-cell and cell-substrate

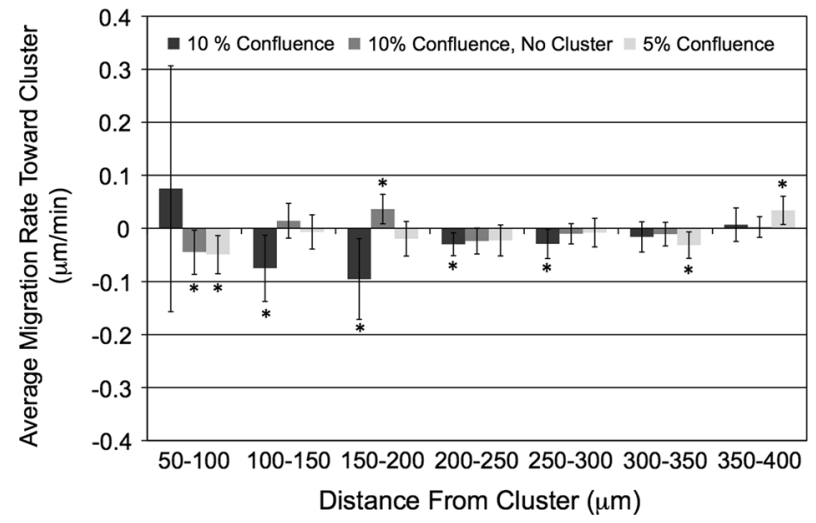

FIGURE 3. Average migration rate. Average migration rates toward (positive values) or away from (negative values) the cluster as a function of distance from the cluster. Error bars represent the $95 \%$ confidence interval. adhesion strengths were modeled as probabilities and were the primary independent variables in the model. Clustering was quantified with three measurements: (1) average cluster size, (2) average height (3-dimensionality), and (3) compactness (packing efficiency). Three measurements were necessary because just one does not adequately characterize the state of the system. For example, a cluster that is large could be tall and compact, short and spread, or a patchy monolayer of cells. Average height and compactness are metrics that serve to differentiate between these different manifestations of clusters.

To help describe the predominant effects of cellsubstrate and cell-cell adhesion probabilities and demonstrate the diversity of observed results, a few selected examples will be discussed. At $0 \%$ cell-substrate probability, the least degree of clustering occurred at $0 \%$ cell-cell adhesion probability (Fig. 5b). Increasing cell-cell adhesion probability to $50 \%$ only modestly increased average cluster size, height, and compactness (Fig. 5c). Values for average height and compactness peaked at $90 \%$ cell-cell adhesion probability (Fig. 5d) then declined as cell-cell adhesion probability approached 99\% (Fig. 5e). However, average cluster size continued to increase up to the
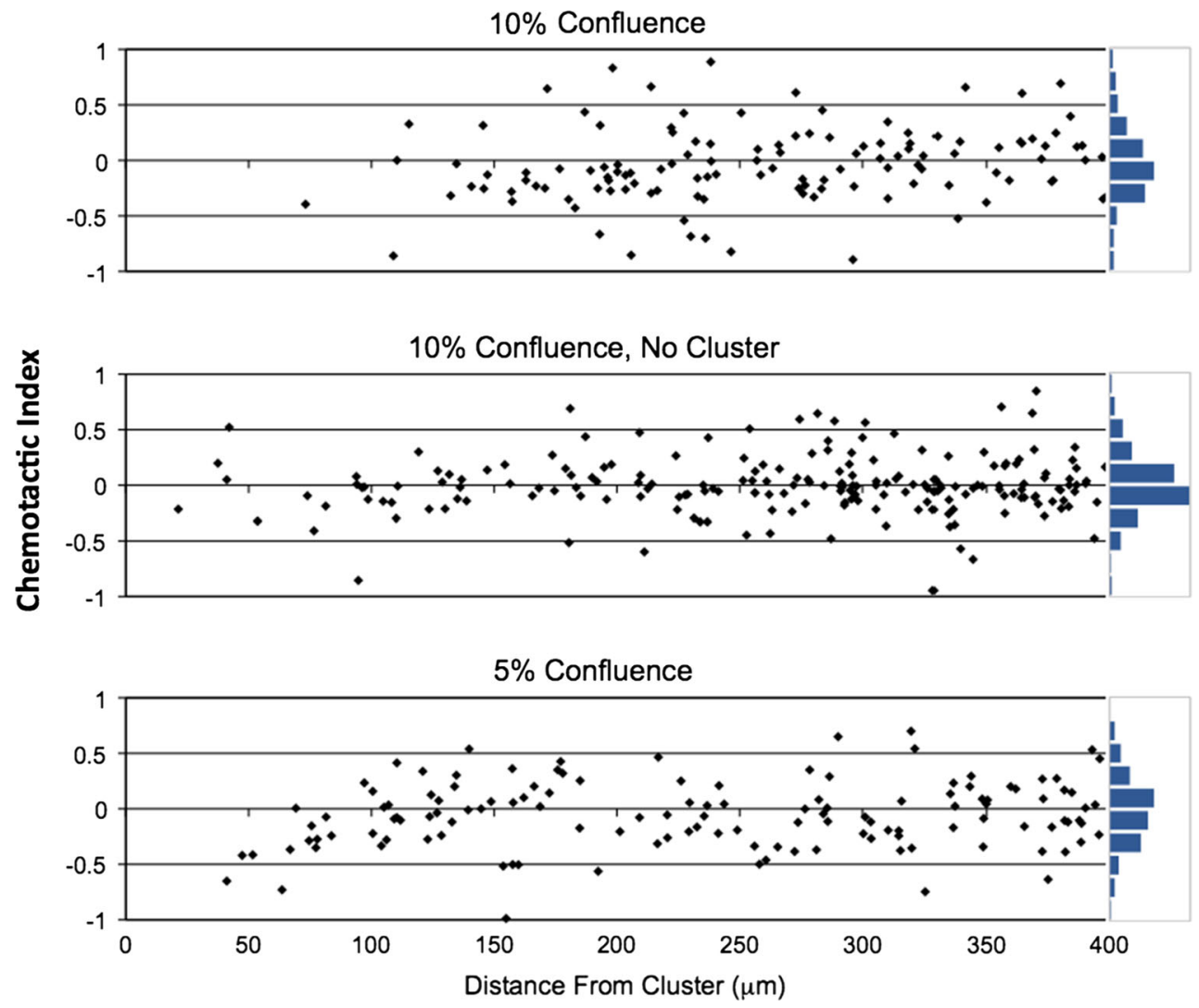

FIGURE 4. Chemotactic index values. Chemotactic index values for all cell tracks as a function of distance from the cluster. The histogram represents the number of points within each 0.2 chemotactic index range. 

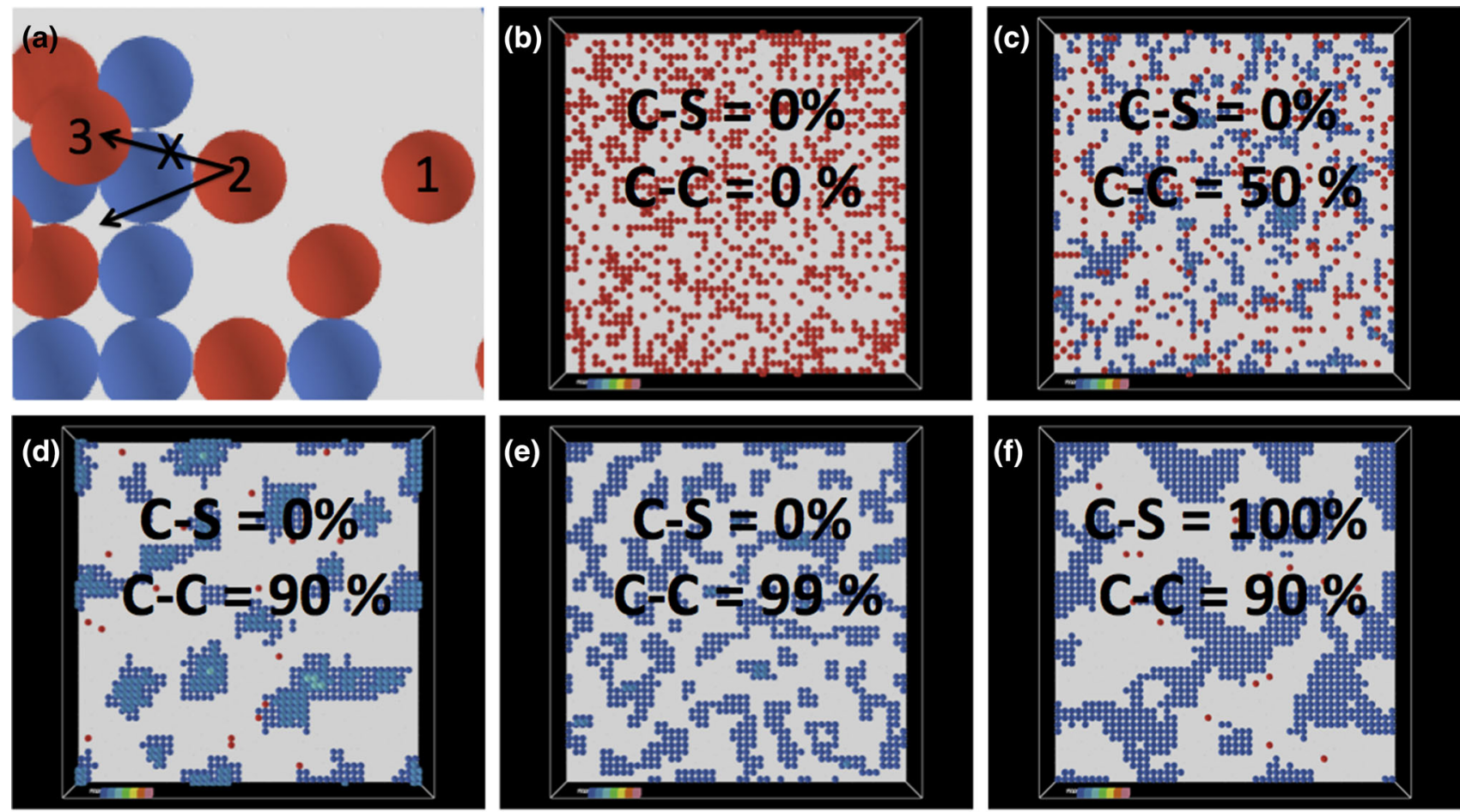

(g)

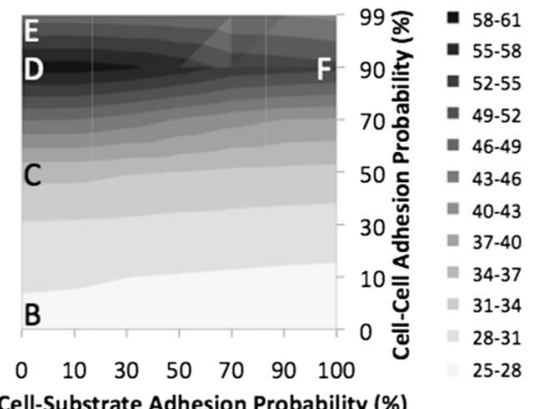

(h)

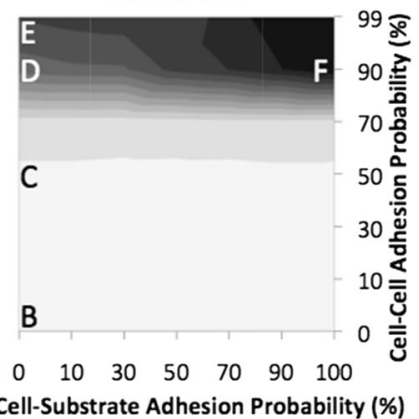

(i)

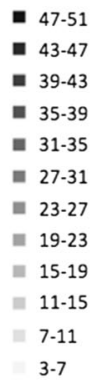

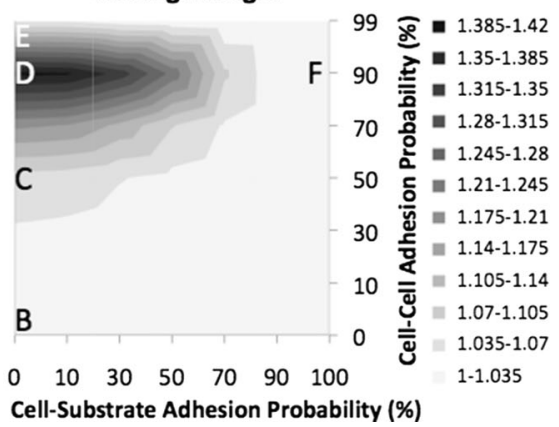

FIGURE 5. Agent-based model simulation results. (a-f) Unbound cells possess no cell-cell bonds, are labeled red, and may move within the simulation space to unoccupied neighboring sites. Cells that possess at least one cell-cell bond are color-coded depending upon the level they occupy from dark blue on the base level to pink at the uppermost level. Cells on successive levels are offset 0.5 patches in the $X$ and $Y$ directions. (a) As examples, cell one has no neighbors and therefore its movement is unrestricted. Cell two may move freely within its current plane up, right and down. Depending on the cell-substrate adhesion probability, cell two may also move left by climbing to one of two potential locations (arrows). Since there is no substrate above the base layer, to climb, these locations must be supported by four cells and be unoccupied. Four cells support both leftward locations, but one of these locations is occupied by cell three leaving just one location that cell two may climb to. Cell three is an unbound cell on level two with no neighbors. If cell three moves down, it will remain on level 2. If cell three were to move right it would no longer be supported by four cells and would fall to level 1. (b-f) Images from a selection of simulations after reaching equilibrium that demonstrate the diversity of observed results. Cell-cell adhesion probability (C-C) and cell-substrate adhesion probability (C-S) are labeled on each panel. Clustering was quantified using three measurements: cluster size (G), compactness $(\mathrm{H})$, and average height (I). Data are presented as topographic plots and labeled to highlight the data corresponding to panels (b-f). For scale, cell diameters are $20 \mu \mathrm{m}$.

maximum value for cell-cell adhesion probability. Regardless of cell-cell adhesion probability, as cellsubstrate adhesion probability was increased cells were increasingly inhibited from climbing. This resulted in the formation of clusters that were flatter and less compact, but larger as clusters merged together (Fig. 5f).
Simulation results reveal that both cell-cell and cellsubstrate adhesion probabilities influence the presence and extent of cell clustering (Fig. 5). Consistent with the explanation provided by differential adhesion, increased clustering occurs at higher cell-cell adhesion probabilities. At these higher cell-cell adhesion probabilities, low cell-substrate adhesion favors the devel- 
opment of clusters that are thick (in the $\mathrm{z}$ direction) and compact (few void spaces), while high cell-substrate adhesion favors large interconnected patches of cells in a monolayer (Figs. 5g-5i).

Though most combinations of cell-cell and cellsubstrate adhesion probability did not support the formation of clusters, the optimal 3D clusters formed with the minimum value for the cell-substrate adhesion probability and a cell-cell adhesion probability between 70 and 99\% (Supplementary Movie S4). Clustering was more pronounced (i.e., larger, thicker clusters) at these same conditions when confluence was increased to $75 \%$ (Supplementary Movie S5). These results suggest that that differential adhesion is a plausible mechanism by which PANC-1 cells might cluster in the absence of other influences.

\section{CONCLUSION}

This study may be the first study to track single PANC-1 cell migration during conditions that support clustering. In our analyzes, we did not observe directed migration in any of our experiments. Instead, our results are consistent with previous examples of cells moving in persistent random walks. ${ }^{32}$ Additionally, our agentbased model based on differential adhesion demonstrated that clustering can occur in the absence of longrange interactions and identified certain conditions that are more favorable toward the generation of three-dimensional clusters. A low cell-substrate adhesion probability and high cell-cell adhesion probability allowed clusters to form that were of moderate size, round, and 3D. Although these in vitro experiments did not identify a mechanism for PANC-1 clustering, the computational experiments give credence to alternate possibilities such as differential adhesion.

Together, our experimental and computational results can provide some suggestions for researchers trying to facilitate the clustering of cells in vitro. Though a moderately adhesive surface is known to support the greatest rates of cell migration, ${ }^{11}$ consequently increasing the frequency of cell-cell collisions, an overly adhesive surface will inhibit the formation of $3 \mathrm{D}$ clusters by not allowing cells to detach. Therefore, the choice of substrate and cell culture media should be carefully considered for their impact on cell-substrate adhesion. In addition, our computational results suggest that the relative strength of cell-cell and cellsubstrates influenced not just the size of the cluster but its compactness and average height, which could potentially modulate differentiation toward endocrine phenotype. The cumulative knowledge learned from this and other PANC-1 cell models will likely extend to therapeutic cell lines and could potentially increase pancreatic endocrine differentiation and the production of hormones by these cells for the treatment of type I diabetes.

\section{ELECTRONIC SUPPLEMENTARY MATERIAL}

The online version of this article (doi: 10.1007/s12195-015-0396-5) contains supplementary material, which is available to authorized users.

\section{ACKNOWLEDGMENTS}

This work was supported by National Science Foundation Grants NSF-CMMI (0928739 and 1334757).

\section{CONFLICT OF INTEREST}

Mr. Holfinger and Drs. Reinhardt, Reen, Schultz, Passino, Ackerman, Kniss, Sander, Gallego-Perez, and Gooch have no conflicts of interest.

\section{ETHICAL STANDARDS}

No human or animal studies were carried out by the authors for this article.

\section{REFERENCES}

${ }^{1}$ Bauwens, C. L., et al. Control of human embryonic stem cell colony and aggregate size heterogeneity influences differentiation trajectories. Stem Cells 26:2300-2310, 2008.

${ }^{2}$ Beattie, G. M., et al. A novel approach to increase human islet cell mass while preserving beta-cell function. Diabetes 51:3435-3439, 2002.

${ }^{3}$ Berens, P. CircStat: A MATLAB toolbox for circular statistics. J. Stat. Softw. 31:1-21, 2009.

${ }^{4}$ Bonner-Weir, S., et al. In vitro cultivation of human islets from expanded ductal tissue. Proc. Natl. Acad. Sci. USA 97:7999-8004, 2000.

${ }^{5}$ Boretti, M. I., and K. J. Gooch. Induced cell clustering enhances islet beta cell formation from human cultures enriched for pancreatic ductal epithelial cells. Tissue Eng. 12:939-948, 2006.

${ }^{6}$ Boretti, M. I., and K. J. Gooch. Effect of extracellular matrix and 3D morphogenesis on islet hormone gene expression by Ngn3-infected mouse pancreatic ductal epithelial cells. Tissue Eng. A 14:1927-1937, 2008.

${ }^{7}$ Brereton, H. C., et al. Homotypic cell contact enhances insulin but not glucagon secretion. Biochem. Biophys. Res. Commun. 344:995-1000, 2006.

${ }^{8}$ Choi, Y. Y., B. G. Chung, D. H. Lee, A. Khademhosseini, J.-H. Kim, and S.-H. Lee. Controlled-size embryoid body formation in concave microwell arrays. Biomaterial 31:4296-4303, 2010. 
${ }^{9}$ Curtis, A. S., and J. V. Forrester. The competitive effects of serum proteins on cell adhesion. J. Cell Sci. 71:17-35, 1984.

${ }^{10}$ Davis, G. E., and C. W. Camarillo. Regulation of endothelial cell morphogenesis by integrins, mechanical forces, and matrix guidance pathways. Exp. Cell Res. 216:113-123, 1995.

${ }^{11}$ DiMilla, P. A., J. A. Stone, J. A. Quinn, S. M. Albelda, and D. A. Lauffenburger. Maximal migration of human smooth muscle cells on fibronectin and type IV collagen occurs at an intermediate attachment strength. J. Cell Biol. 122:729-737, 1993.

${ }^{12}$ Ferrell, N., et al. Vacuum-assisted cell seeding in a microwell cell culture system. Anal. Chem. 82:2380-2386, 2010.

${ }^{13}$ Gan, M. J. A. Albanese-O'Neill, and M.J. Haller. Type 1 diabetes: current concepts in epidemiology, pathophysiology, clinical care, and research. Curr. Probl. Pediatr. Adolesc. Health Care 42:269-291, 2012.

${ }^{14}$ Gershengorn, M. C., A. A. Hardikar, C. Wei, E. GerasRaaka, B. Marcus-Samuels, and B. M. Raaka. Epithelialto-mesenchymal transition generates proliferative human islet precursor cells. Science 306:2261-2264, 2004.

${ }^{15}$ Green, J. E. F., S. L. Waters, K. M. Shakesheff, and H. M. Byrne. A mathematical model of liver cell aggregation in vitro. Bull. Math. Biol. 71:906-930, 2009.

${ }^{16}$ Hansen, C. H., R. G. Endres, and N. S. Wingreen. Chemotaxis in Escherichia coli: a molecular model for robust precise adaptation. PLoS Comput. Biol. 4:e1, 2008.

${ }^{17}$ Hardikar, A. A., B. Marcus-Samuels, E. Geras-Raaka, B. M. Raaka, and M. C. Gershengorn. Human pancreatic precursor cells secrete FGF2 to stimulate clustering into hormone-expressing islet-like cell aggregates. Proc. Natl. Acad. Sci. USA 100:7117-7122, 2003.

${ }^{18}$ Hatziavramidis, D. T., T. M. Karatzas, and G. P. Chrousos. Pancreatic islet cell transplantation: an update. Ann. Biomed. Eng. 41:469-476, 2013.

${ }^{19}$ Hummel, K., K. K. McFann, J. Realsen, L. H. Messer, G. J. Klingensmith, and H. P. Chase. The increasing onset of type 1 diabetes in children. J. Pediatr. 161:652-657, 2012.

${ }^{20}$ Hwang, Y.-S., B. G. Chung, D. Ortmann, N. Hattori, H.C. Moeller, and A. Khademhosseini. Microwell-mediated control of embryoid body size regulates embryonic stem cell fate via differential expression of WNT5a and WNT11. Proc. Natl. Acad. Sci. USA 106:16978-16983, 2009.

${ }^{21}$ James, S. A. M., et al. Islet transplantation in seven patients with type 1 diabetes mellitus using a glucocorticoidfree immunosuppressive regimen. N. Engl. J. Med. 343:230-238, 2000.

${ }^{22}$ Kay, R. R., P. Langridge, D. Traynor, and O. Hoeller. Changing directions in the study of chemotaxis. Nat. Rev. Mol. Cell Biol. 9:455-463, 2008.

${ }^{23}$ LeCluyse, E. L., P. L. Bullock, and A. Parkinson. Strategies for restoration and maintenance of normal hepatic structure and function in long-term cultures of rat hepatocytes. Adv. Drug Deliv. Rev. 22:133-186, 1996.

${ }^{24}$ Luther, M. J., et al. MIN6 beta-cell-beta-cell interactions influence insulin secretory responses to nutrients and non-nutrients. Biochem. Biophys. Res. Commun. 343:99-104, 2006.
${ }^{25} \mathrm{Ma}$, X., et al. Fibers in the extracellular matrix enable longrange stress transmission between cells. Biophys. J. Biophys. Soc. 104:1-9, 2013.

${ }^{26}$ Maher, J., J. V. Martell, B. A. Brantley, E. B. Cox, J. E. Niedel, and W. F. Rosse. The response of human neutrophils to a chemotactic tripeptide ( $N$-formyl-methionylleucyl-phenylalanine) studied by microcinematography. Blood 64:221-228, 1984.

${ }^{27}$ Mishra, P. K., S. R. Singh, I. G. Joshua, and S. C. Tyagi. Stem cells as therapeutic target for diabetes. Front Biosci. 15:461-477, 2011.

${ }^{28}$ Park, J., et al. Microfabrication-based modulation of embryonic stem cell differentiation. Lab Chip 7:1018-1028, 2007.

${ }^{29}$ Pictet, R. L., W. R. Clark, R. H. Williams, and W. J. Rutter. An ultrastructural analysis of the developing embryonic pancreas. Dev. Biol. 29:436-467, 1972.

${ }^{30}$ Ramiya, V. K., M. Maraist, K. E. Arfors, D. A. Schatz, A. B. Peck, and J. G. Cornelius. Reversal of insulin-dependent diabetes using islets generated in vitro from pancreatic stem cells. Nat. Med. 6:278-282, 2000.

${ }^{31}$ Rosenberg, L. Induction of islet cell neogenesis in the adult pancreas: the partial duct obstruction model. Microsc. Res. Tech. 43:337-346, 1998.

${ }^{32}$ Saltzman, W. M. Tissue engineering: engineering principles for the design of replacement organs and tissues. New York: Oxford University Press, p. 544, 2004.

${ }^{33}$ Shapiro, A. M. J., et al. International trial of the Edmonton protocol for islet transplantation. N. Engl. J. Med. 355:1318-1330, 2006.

${ }^{34}$ Steinberg, M. S. Reconstruction of tissues by dissociated cells. Science 141:401-408, 1963.

${ }^{35}$ Van Haastert, P. J. M., and M. Postma. Biased random walk by stochastic fluctuations of chemoattractant-receptor interactions at the lower limit of detection. Biophys. J. 93:1787-1796, 2007.

${ }^{36}$ Vernon, R. B., J. C. Angello, M. L. Iruela-Arispe, T. F. Lane, and E. H. Sage. Reorganization of basement membrane matrices by cellular traction promotes the formation of cellular networks in vitro. Lab. Invest. 66:536-547, 1992.

${ }^{37}$ Wei, C., E. Geras-Raaka, B. Marcus-Samuels, Y. Oron, and M. C. Gershengorn. Trypsin and thrombin accelerate aggregation of human endocrine pancreas precursor cells. J. Cell. Physiol. 206:322-328, 2006.

${ }^{38}$ Widman, M. T., D. Emerson, C. C. Chiu, and R. M. Worden. Modeling microbial chemotaxis in a diffusion gradient chamber. Biotechnol. Bioeng. 55:191-205, 1997.

${ }^{39}$ Wilensky, U. NetLogo. http://ccl.northwestern.edu/netlogo/. Center for Connected Learning and Computer-Based Modeling, Northwestern University. Evanston, IL: Center for Connected Learning and Computer-Based Modeling, Northwestern University, 1999.

${ }^{40}$ Winer, J. P., S. Oake, and P. A. Janmey. Non-linear elasticity of extracellular matrices enables contractile cells to communicate local position and orientation. PLOS ONE 4:e6382, 2009. 